\title{
Preface
}

\section{Shoulder Arthritis in the Young and Active Patient}

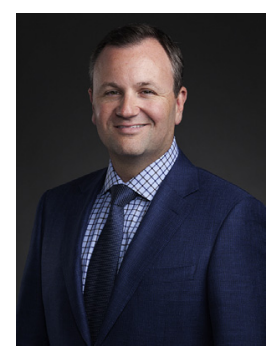

Stephen F. Brockmeier, MD

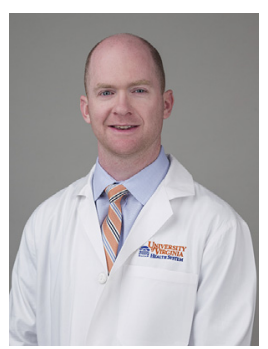

Brian C. Werner, MD

Editors

Shoulder arthritis is common, and management strategies, especially in "young" patients, continue to evolve. While shoulder arthroplasty is a reliable option for treatment of the entity in older, less active patients, there is not a clear management pathway in patients who develop arthritis at a younger age. There have been significant improvements in implant design, but longevity remains a concern in more active patients, leading to the treatment challenge and the focus of this issue of Clinics in Sports Medicine. Why does shoulder arthritis occur at a young age, are there any reliable surgical treatments other than arthroplasty in this age group, and if arthroplasty is the only option, will younger patients be able to remain active? We confront these questions and many others addressed in this issue commonly in all of our practices.

The issue opens with a comprehensive review of the cause of glenohumeral arthritis in young individuals, which include instability, trauma, genetic causes, and surgical complications among others. This leads nicely into an excellent summary by Dr Petrigilano and colleagues about nonarthroplasty options for young individuals with shoulder arthritis, such as viscosupplementation, arthroscopic debridement, microfracture, osteochondral allograft, and interposition grafts. Additional biologic options for this entity are described in a subsequent article by Dr Goel and coauthors. Several articles are then dedicated to describing the use of arthroplasty in the young and active population, including both hemiarthroplasty and total shoulder arthroplasty, rehabilitation protocols, and expected outcomes, including return to activities and sports. We conclude with an article by Drs Tashjian and Chalmers describing the future frontiers in shoulder arthroplasty.

We would like to thank the editor and editorial staff of Clinics in Sports Medicine for the invitation to put together a comprehensive update on this area and would like to 
sincerely thank all of the authors who contributed their expertise and effort to the development of this issue.

Stephen F. Brockmeier, MD Department of Orthopaedic Surgery University of Virginia 400 Ray C. Hunt Drive, Suite 330 Charlottesville, VA 22903, USA

Brian C. Werner, MD Department of Orthopaedic Surgery University of Virginia 400 Ray C. Hunt Drive, Suite 330 Charlottesville, VA 22903, USA E-mail addresses: sfb2e@virginia.edu (S.F. Brockmeier) Bcw4x@virginia.edu (B.C. Werner) 